\title{
Segmenting Images like MR Brain, Breast and Scintigraphy Thyroid Gland using Fuzzy C Means Based Morphological Reconstruction Filters
}

\author{
R.Sumathi, M.Venkatesulu
}

\begin{abstract}
Our study has introduced a new modified methodology using Fuzzy C Means clustering with morphological reconstruction filters to segment the abnormal parts in multimodal images such as MR brain, MR breast and scintigraphy thyroid gland.MR scanning is helpful to analyze the internal behavior of the tumor, whereas scintigraphy scanning is used to analyze the shape and location of the gland and also prevent the cancerous stage. We have used samples from public dataset like Harvard brain dataset for the brain, RIDER for breast and TCGA - THCA for the thyroid gland. In the first step, we preprocessed the image by applying the median filter which removes the noisy information present in the given input image. In the second step, Fuzzy C Means clustering was used to segment the boundary of the abnormal part in the multimodal images. In the last step, morphological reconstruction filters are used to segment the accurate shape and location of the abnormal part in all the three multimodal images. The performance and the efficiency of the segmentation were computed using the measures such as entropy, eccentricity, MSE, PSNR, sensitivity, specificity, accuracy and computational time. The results from our modified method show an accurate segmentation for all multimodalities images within $4 m s$ and its accuracy rate is nearly $95 \%$ for all types of images when compared with existing techniques such as $K$-means and $G A$ with $K$ - Means. A new modified method using Fuzzy $C$ means clustering with morphological reconstruction filters was applied to segment the abnormal part accurately with minimum duration in all multimodal images
\end{abstract}

Keywords : Image Segmentation, Median Filter, Fuzzy C Means Clustering, Performance Measures

\section{INTRODUCTION}

$\mathrm{M}$ edical image processing has become an important part of clinical diagnosis but contrast, noise and other factors can affect the quality of images. Many studies report for the importance of segmentation in the medical field, and extraction of the region of interest for various diagnostic applications. segmentation plays a vital role in detecting the dynamic behavior of various diseases. Mathematical morphology is very powerful for all biomedical images.

Revised Manuscript Received on July 22, 2019.

* R.Sumathi

R.Sumathi*, Department of Computer Science and Engineering, Kalasalingam Academy of Research and Education, Tamil Nadu, India. Email: suchandika@gmail.com

M.Venkatesulu, Department of Information Technology,

Kalasalingam Academy of Research and Education, Tamil Nadu, India

Email: venkatesulu2000m@gmail.com
Image segmentation work is to segment the given image into number of smaller regions depending on the features like color, texture etc[1]. Kaur and Jindal [2] used various segmentation methods like active contour without edges, localized active contour without edges and distance regularized level set. These techniques have been chosen carefully based on the physiological relevance to the applications in order to identify the part of interest. It is important to minimize the high frequency component without any loss of information, but median filters are suitable for reducing much blur information than any other filters reported by [3-4]. Agustin and Babu [5] applied the following techniques for the segment the thyroid gland in images. Kaur and Wasson [6] applied neural network and segment thyroid nodules on ultrasound images. Localized based algorithm was applied by Lankton and Tannenbaum [7] which is used to select the portion of thyroid gland based on the location of thyroid and segment that part, and able to classify the nodule whether it is benign and malignant. Dubey and Mushrif [8] applied various methods with standard fuzzy objective function and compare its features and analyze all the issues for improving the accurate segmentation [9]. Features extraction with heuristic algorithm is proposed to segment the tumor part with $6150.635154 \mathrm{~ms}$ time with 0.906348 of entropy value. Alomoush et al [10] analyzed various segmentation approach with FCM and with FA,PSO,HAS etc and discussed the advantages and issues in detail[11]. FCM based SVM classifier is applied to segment the brain tumor whereas FCM is used for tumor segmentation and SVM is used for classify cancer or non cancer by Kaur and Reecha [12] utilized FCM clustering for brain tumor segmentation with region growing algorithm and proved that sobel edge detector is best for tumor segmentation and performance is ensured with Relative Ultimate Measurement Accuracy and standard deviation. Vijayalakshmi et al [13] used FCM segmentation for segmenting the masses in breast mammogram images. [14] A semi supervised segmentation approach is used to segment and classify the breast tumor with limited parameters used and ensured the accuracy with various state of art. Vesal et al [15] applied automatic watershed segmentation to segment breast lesions of MR breast images. 


\section{Segmenting Images like MR Brain, Breast and Scintigraphy Thyroid Gland using Fuzzy C Means Based Morphological Reconstruction Filters}

\section{METHODS AND MATERIALS}

The aim of the study is to apply a new modified single modality to segment the timorous part with minimum duration without changing the FCM parameter for all images. This analysis is useful for all researchers in future to enhance their knowledge and tried a single method for many modalities. This system is helpful for the radiologist to measure the depth of diseases in advance. we compare the segmentation accuracy and computation time with K Means clustering and GA with $\mathrm{K}$ Means clustering techniques

\subsection{Details of Image datasets}

For our study and analyze the mulmodal image segmentation we have ised various image datasets available through online and some from clinical data set for proving the efficiency of our methodology.

\subsubsection{Scintigraphy Thyroid}

Thyroid scintigraphy is a procedure producing more planar images of thyroid obtained within 15-30min after intravenous injection of Tc-99 was mentioned in [16]. Scintigraphy images can also detect abnormal but functional thyroid tissue by virtue of its iodine/analogue concentrating ability. Scintigraphy is a nuclear medicine test that allows the doctor to determine the structure, shape and correct location of the thyroid gland. The thyroid gland looks like a butterfly in shape.It is found at the front of the neck. It secretes the hormones and regulates the rate of metabolism. The gland carries hormones to all tissues in the body[16-17]

\subsubsection{MR brain images}

MRI scanning uses a magnetic field and radio waves which clearly capture the detailed information from the brain images. Based on the scan report radiologist measure the depth of tumor and prevent the severity in early stage. T1 and T2 axial images are used for our study. All MR brain images were collected from publicly avaliable sources including Harvard brain dataset, BRATS dataset and clinical data sets.

\subsubsection{MR Breast images}

Nowadays many womens in the world are highly affected by breast cancer and inceases the death rate. We collected the post processed, T1 post contrast images from clicnical dataset and some images from online source like Reference Image Database to Evaluate Therapy Response (RIDER) and Breast Imaging Reporting and Database System (BIRADS)[18]

\section{Image Analysis}

The images were analyzed using the following steps

Step 1: Read all multimodal images and resize into $256 * 256$ grayscale images

Step 2: Preprocess the image by applying the median filters and to segment the boundary of tumor part in multimodal images using Fuzzy C Means clustering

Step 3: To delete the patches and bright holes in the image by applying an morphological reconstruciton operatioms to measure the affected part of all mutlmodal images which is useful fordiagnosis at early. Figure. 1 shows the flow diagram of our proposed work.

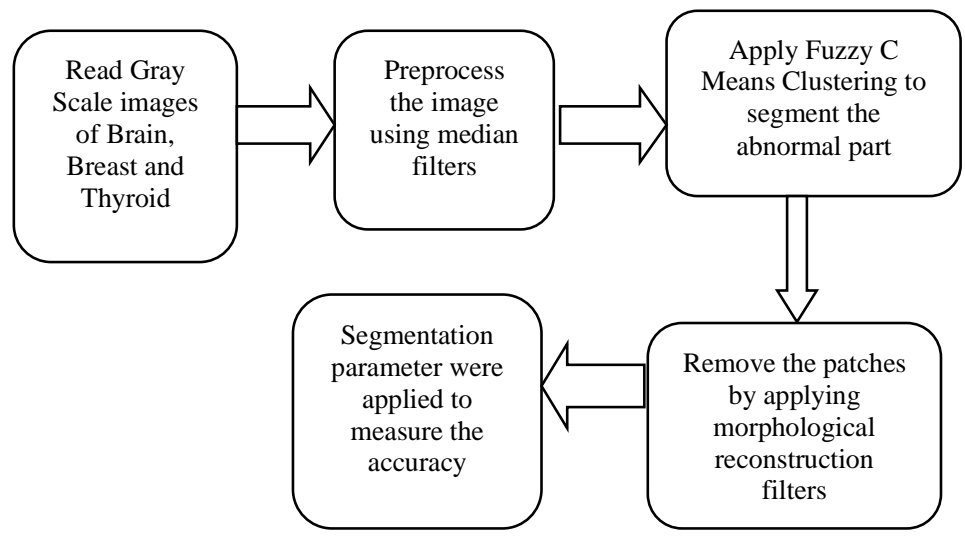

Figure 1 : Flow diagram of our proposed work

\subsection{Median Filtering}

Median filtering was applied to reduce the noise information which appears due to the presence of minute gray scale variation in the image. Median filtering is the most used image enhancement technique for removing impulse noise without effectively reducing the image sharpness suggested by [3-4]

$$
\mathrm{f}(\mathrm{x}, \mathrm{y})=\operatorname{median}\{\mathrm{g}(\mathrm{s}, \mathrm{t})\}
$$

where as $f(x, y)$ is the median filtered image and $g(s, t)$ is the original input image

\subsection{Fuzzy C Means Clustering}

FCM algorithm is a clustering technique developed by Dunn, improved by Bezdek and further improved by Matteo Matteucci. Fuzzy C Means (FCM) algorithm is a feasible method suitable for many image segmentation applications. Its special feature is that it retains more information than hard segmentation techniques. It groups the similar pixels based on the number of clusters specified in the input value. From the centroid, least mean distance among all the pixels was calculated and the value of centroid was reassigned as low membership grade. This process was repeated many times to reduce the objective function mentioned in [19-20]

$$
\mathrm{J}_{\mathrm{k}}=\sum_{\mathrm{i}=\mathrm{n}}^{\mathrm{N}} \sum_{\mathrm{j}=1}^{\mathrm{c}} \delta_{\mathrm{ij}}\left\|\mathrm{x}_{\mathrm{i}}-\mathrm{c}_{\mathrm{j}}\right\|{ }^{2}
$$

Where $\mathrm{N}$ represents the number of data points used in input and $\mathrm{K}$ represents the number of iteration being performed, whereas $c_{j}$ gives the centriod value,$x_{i}$ defines the data points, $\delta_{\mathrm{ij}}$ represents the degree of membership for ith data point in $\mathrm{x}_{\mathrm{i}}$ in the cluster $\mathrm{j}$, and $\left\|\mathrm{x}_{\mathrm{i}}-\mathrm{c}_{\mathrm{j}}\right\|$ produces the mean distance between $x_{i}$ and cluster $j$. The membership is shown below 


$$
\delta_{\mathrm{ij}}=\frac{1}{\sum_{\mathrm{k}=1}^{\mathrm{c}}\left[\frac{\left\|\mathrm{x}_{\mathrm{i}}-\mathrm{c}_{\mathrm{j}}\right\|}{\left\|\mathrm{x}_{\mathrm{i}}-\mathrm{c}_{\mathrm{k}}\right\|}\right] \cdot \frac{2}{\mathrm{~m}-1}}
$$

Where $m$ represents the fuzzy coefficient obtanied for the overlapping clusters renging from $1.5 \leq \mathrm{m} \leq 2.5$ for optimal segmentation, whereas center vector for any clutser can be defined as

$$
\mathrm{c}_{\mathrm{j}}=\frac{\sum_{\mathrm{i}-1}^{\mathrm{N}} \delta_{\mathrm{ij}} * \mathrm{x}_{\mathrm{i}}}{\sum_{\mathrm{i}-1}^{\mathrm{N}} \delta_{\mathrm{ij}}}
$$

\subsection{Morphological Reconstruction Filters}

The morphological reconstruction filter is a very helpful methodology to smoothening the noisy gray-level images by a determined composition of opening and closing with a given structural element [21] (defined by Serra (1982). It preserves the contours with the help of both opening and closing operator and it is applied to the input image $\mathrm{A}$ and the structuring element B represented by [22] Rafael C. Gonzalez

An opening operator is defined as

$$
\mathrm{A}^{\circ} \mathrm{B}=(\mathrm{A} \ominus \mathrm{B}) \oplus \mathrm{B}
$$

Opening by reconstruction filter operator helps to segment the exact shape of abnormal part efficiently in all medical images. Closing operator is represented as

$$
\mathrm{A} \cdot \mathrm{B}=(\mathrm{A} \oplus \mathrm{B}) \ominus \mathrm{B}
$$

Both opening and closing is useful for medical imaging to remove the local gray intensity value without changing the borders of regions. In this study we use disc as the structural element and its size is fixed as 7X7 for all modalities.

\section{III .RESULTS AND DISCUSSION}

Segmentation metrics like Mean Square Error (MSE), peak Signal to Noise (PSNR), segmentation accuracy and computation time are measured to ensure accuracy of our proposed work. It was implemented by using MATLAB 2017a software with Intel Core i5 processor of 4GB RAM. In our method, we used $\mathrm{N}=2$ and $\mathrm{K}=20$ for Fuzzy $\mathrm{C}$ Means Clustering technique for boundary segmentation and for removing unwanted pixels by applying the structural element.

The Mean square Error is denoted by

$M S E=\frac{\sum M, N\left[x_{1}(m, n)-x_{2}(m, n)\right] \cdot^{2}}{M * N}$

$\mathrm{M}$ and $\mathrm{N}$ represents the row and column used in the given input image. Whereas peak signal to noise Ratio can be calculated by

$$
P S N R=10 \log _{10}\left(\frac{R^{2}}{M S E}\right)
$$

Where $\mathrm{R}$ represents the maximum fluctutation value of the given imput image. It measures the quality of noise information between the input and segmented image computational time is measured between the given images and extracted output thyroid gland image. Sensitivity measures the area of thyroid gland from the total area of the image

Sensitivity $=\frac{T_{p}}{T_{p}+F_{n}}$

Specificity measures the background ratio from the total area of the background

Specivicity $=\frac{T_{n}}{T_{n}+F_{p}}$

Accuracy represents the segmentation rate between the manual segmentation and systematically segmentation

$$
\text { Accuracy }=\frac{T_{p}+T_{n}}{T_{p}+T_{n}+F_{p}+F_{n}}
$$

Segmentation metrics like MSE, PSNR for all three modalities with existing approach like $\mathrm{K}$ means and GA with $\mathrm{K}$ means clustering is shown in Table 1 . From the results, it seems that MSE values are increasing and PSNR values are decreasing for the entire tested image, and the average value of PSNR is 34.15 .

Table 1: MSE and PSNR comparison among K means, GA with $\mathrm{K}$ means and FCM with morphological reconstruction filters

\begin{tabular}{|c|c|c|c|c|c|c|c|c|c|}
\hline \multicolumn{9}{|c|}{ K Means Clustering } & \multicolumn{3}{l|}{ GA with K Means Clustering } & \multicolumn{3}{l|}{ Fuxy C Means Clustering } \\
\hline Img & MSE & PSNR & Time (s) & MSE & PSNR & Time (s) & MSE & PSNR & Time (s) \\
\hline 1 & 34.1 & 38.87 & 3.89 & 0.038 & 28.48 & 4.02 & 0.021 & 42.1 & 3.02 \\
\hline 2 & 42.15 & 37.85 & 5.71 & 66.45 & 24.58 & 5.89 & 0.041 & 33.59 & 3.38 \\
\hline 3 & 40.15 & 30.01 & 4.32 & 0.04 & 78.56 & 4.88 & 0.09 & 35.45 & 3.01 \\
\hline 4 & 34.45 & 38.78 & 3.89 & 21.54 & 34.89 & 5.89 & 0.001 & 47.71 & 3.31 \\
\hline 5 & 65.16 & 32.89 & 6.45 & 76.88 & 54.22 & 7.82 & 0.031 & 32.05 & 3.87 \\
\hline 6 & 44.56 & 31.04 & 9.3 & 65.71 & 39.01 & 6.97 & 0.012 & 31.5 & 2.76 \\
\hline 7 & 48.21 & 30.22 & 7.01 & 53.51 & 28.71 & 8.5 & 0.066 & 28.31 & 3.71 \\
\hline 8 & 65.16 & 32.89 & 5.45 & 67.12 & 33.77 & 6.01 & 48.34 & 30.66 & 5.92 \\
\hline 9 & 67.02 & 33.06 & 6.57 & 58.32 & 42.54 & 6.88 & 23.88 & 31.21 & 4.97 \\
\hline 10 & 72.76 & 33.68 & 5.48 & 98.43 & 20.09 & 6.54 & 24.22 & 31.22 & 4.73 \\
\hline 11 & 73.23 & 33.39 & 7.03 & 87.44 & 44.03 & 7.98 & 21.11 & 31.87 & 6.76 \\
\hline Ave & $\mathbf{5 3 . 3 6}$ & $\mathbf{3 3 . 8 3}$ & 5.92 & 54.13 & $\mathbf{3 8 . 9 9}$ & 6.49 & 10.71 & 34.15 & 4.13 \\
\hline
\end{tabular}

Table 2 represents the accuracy of segmentation with various performance measures like sensitivity, specificity and accuracy. From this table it was ensured that FCM segmentation is suitable for multimodal images. 
Table 2 : Performance Measures of Fuzzy C-Means Clustering

\begin{tabular}{|c|c|c|c|}
\hline Image No. & Sensitivity & Specificity & Accuracy \\
\hline 1 & 94.2 & 99 & 96.5 \\
\hline 2 & 96.7 & 97.3 & 97.5 \\
\hline 4 & 91.9 & 98.5 & 94.9 \\
\hline 5 & 97.2 & 97 & 97.1 \\
\hline 6 & 99 & 96.1 & 97.5 \\
\hline 7 & 91.6 & 98.8 & 95.1 \\
\hline 8 & 92.4 & 72.4 & $93 \%$ \\
\hline 9 & 96.1 & 90.1 & $93 \%$ \\
\hline 10 & 97.6 & 83.2 & $94 \%$ \\
\hline 11 & 96.4 & 90.1 & $95 \%$ \\
\hline Avg & $\mathbf{9 5 . 2}$ & $\mathbf{9 2 . 2 9}$ & $\mathbf{9 5 . 2 4}$ \\
\hline
\end{tabular}

Figure 2 contains MR brain images with T1, T2 axial and post contrast images. Fig 2(a) contains input gray scale image and Fig 2(b) contains FCM segmentation images and Fig 2(c) contains K Means Clustering and Fig 2 (d) contains GA with $\mathrm{K}$ means clustering output.

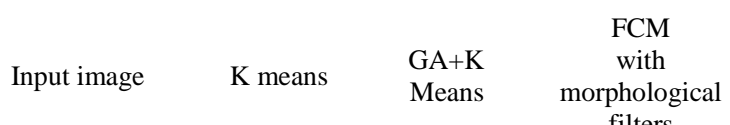
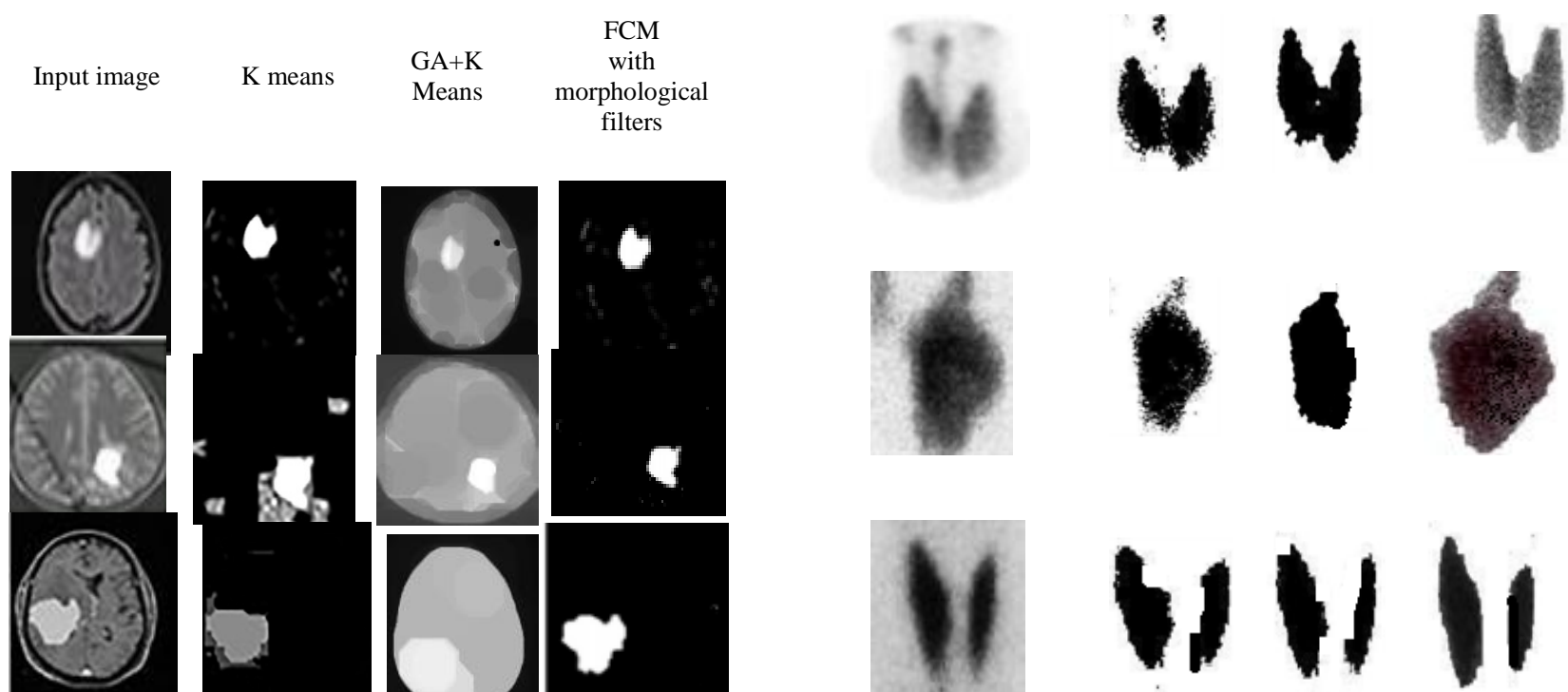

Figure 4 contains Scintigraphy Thyroid gland images includes Iodine 1-3, grave diseases for our validation.
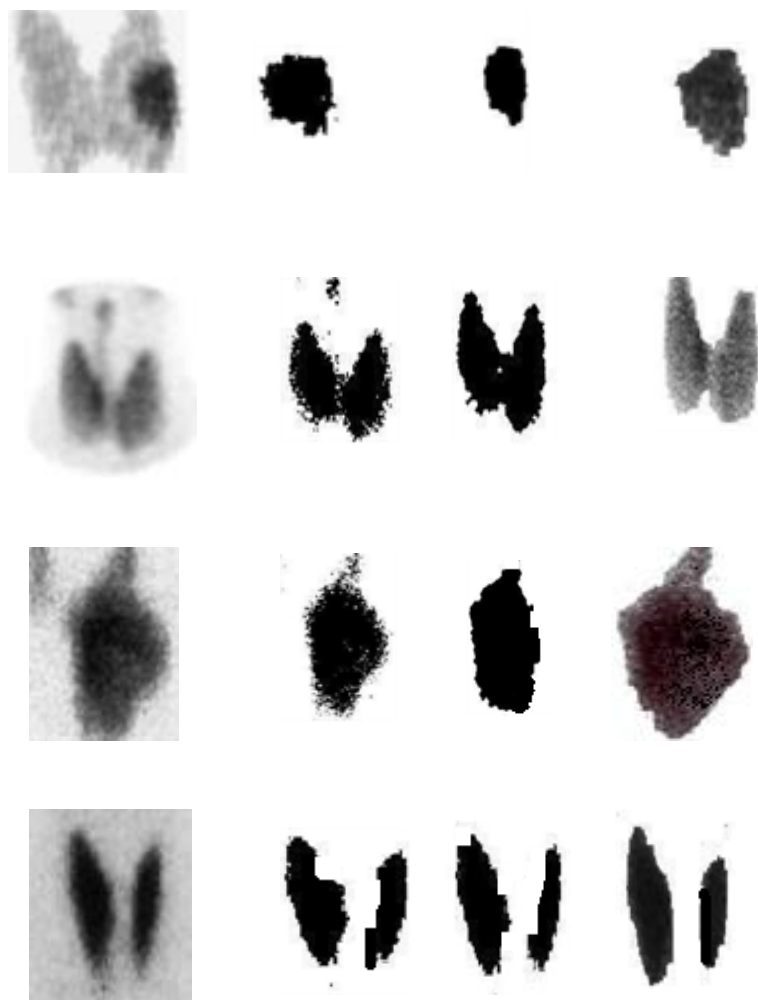

Figure 4 : Scintigraphy Thyroid images with Iodine1-2, Grave diseases

Figure 5 shows the sample ground truth images of MR brain, MR breast and Scintigraphy Thyroid images.
Figure 2: MR brain T1 axial, T2 Axial, T1 post contrast and clinical dataset

Figure 3 contains MR post processed and Post processed contrast breast images with FCM segmentation, K Means Clustering and GA with $\mathrm{K}$ means clustering methods

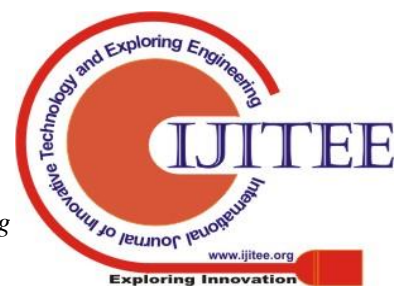



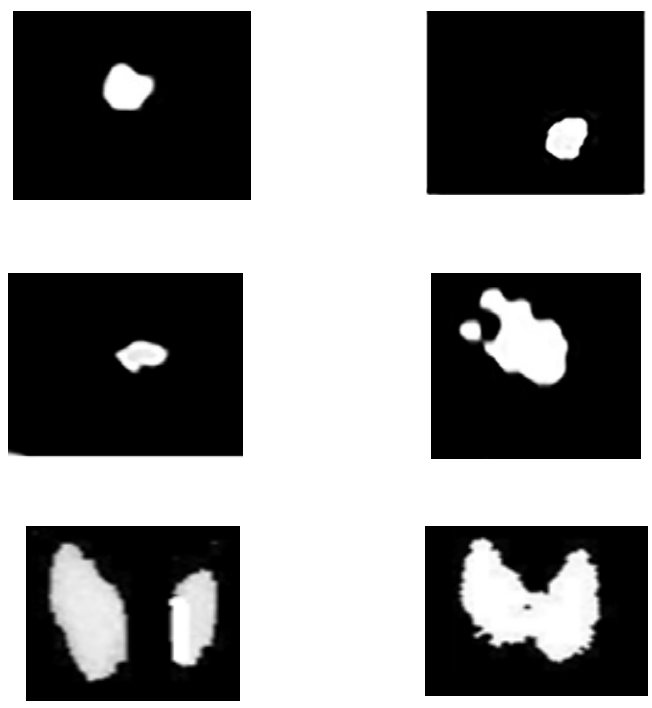

Figure 5: Ground truth images of MR brain, MR breast and scintigraphy thyroid images

Computational time among other techniques are compared with our proposed work is mentioned in Figure 6.

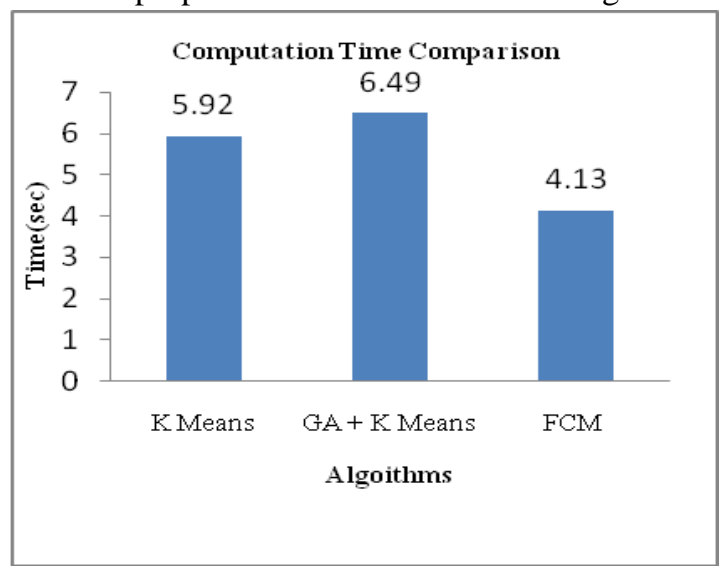

Figure 6: Computational Time among various Techniques with

our proposed work

From all the three modalities segmented output it was proved that a unified algorithm like FCM with morphological reconstruction filters is also suitable for segmenting various medical images within $4 \mathrm{~ms}$

\section{CONCLUSION}

In this work, we have reported a new modified method using FCM with morphological reconstruction filters to segment the tumor present in MR brain, MR breast and scintigraphy thyroid images. The performance metrics such as MSE, PSNR, Sensitivity, Specificity, and Accuracy and Computational time was evaluated and compared the computational time with other state of art methods. Our proposed method segments tumor and thyroid gland within $4 \mathrm{~ms}$ and with average accuracy $95 \%$ which is relatively better than other methods [1,6,23-25]

\section{REFERENCES}

1. M. Roushdy, "Comparative Study of Edge Detection Algorithms Applying on Gray Scale Noisy Image Using Morphological Filters", International Journal on Graphics, Vision and Image processing, 2006, Vol.6, pp.17-23

2. J.Kaur, A.Jindal, "Comparison of Thyroid Segmentation Algorithms in Ultrasound and Scintigrapgy Images", International Journal of Computer Applications. 2012,Vol 50, pp 24-27

3. Ng. PE and Ma. K, "A Switching Median Filter with Boundary Discriminative Noise Detection for Extremely Corrupted Images", IEEE Transaction on Image Processing, 2006, Vol.15, pp.1506-1516

4. R.Yang., Lin. Yin, M.Gabbouj, J.Astola, and Y.Neuvo,."Optimal Weighted Median Filtering Under Structural Constraints”, IEEE Transaction on Signal Processing, 1995, Vol. 43, pp. 591-604.

5. S.A.Agustin, S.Babu, "Thyroid Segmentation on US Medical Images: An Overview". International Journal of Emerging Technology and Advanced Engineering, 2012, Vol. 2, pp 398-404.

6. G.Kaur, V.Wasson," Thyroid Gland Segmentation of Ultrasound Images Based on Neural Networks: A Review", International Journal of Advanced Research in Computer Science and Software Engineering, 2015, Vol. 5, pp. 338-341.

7. S.Lankton, A.Tannenbaum, "A. Localizing Region-Based Active Contours", IEEE Transactions on Image Processing, 2008, Vol.17, pp 2029-2039.

8. Y.K.Dubey M.M. Mushrif, "FCM Clustering Algorithms for Segmentation of Brain MR Images”, Advances in Fuzzy Systems, 2016

9. S.Sasikanth and S.Kumar, "Optimized clustering segmentation using heuristic algorithm (HA) with FCM for brain image analysis", Biomedical Research - Computational Life Sciences and Smarter Technological Advancement. $2^{\text {rd }}$ 2018, pp S124- 132

10. W.Alomoush, A.Alrosan., N.Norwawi, Y.Alomari, D.Albashish, A.Almomani, M.Alqahtania, "Survey: Challenges of Image Segmentation Based Fuzzy C-Means Clustering Algorithm", Journal Of Theoretical And Applied Information Technology, 2008, Vol. 96, pp 5153-70.

11. G.Gupta, V.Singh, "Brain Tumor segmentation and classification using FCM and support vector machine", International Research Journal of Engineering and Technology (IRJET), 2017, Vol. 4, pp.792-96.

12. H.Kaur, Reecha Sharma, "Segmentation of Tumor Region from Brain MRI Images Using Fuzzy C-Means Clustering and Seeded Region Growing”, IOSR Journal of Computer Engineering, 2006, Vol18, pp 20-24

13. S.P.Vijayalakshmi, S.Nair and S.Nithyalakshmi," A FCM Based Approach For Automated Segmentation Of Breast Masses In Mammograms", International Journal of Computational Science and Information Technology (IJCSITY), 2016, Vol.4, pp. 39-47

14. L.Sun, J.He, X Yin, Y Zhang, J-H Chen, T Kron and M-Y Su, “An image segmentation framework for extracting tumors from breast magnetic resonance images", Journal of Innovative Optical Health Sciences, 2018, Vol.11, pp 1-15

15. S.Vesal, A.Diaz-Pinto, N.Ravikumar, S.Ellmann, A.Davari and A.Maier," A, Semi-Automatic Algorithm for Breast MRI Lesion Segmentation Using Marker-Controlled Watershed Transformation”, Conf proc IEEE Nuclear Science Symposium and Medical Imaging Conference, 2017, pp 1-4

16. H.Alshanbari, S.Amain, J.Shuttelworth, K.Slman and M.Muslam, "Automatic Segmentation In Breast Cancer Using Watershed Algorithm”, International Journal of Biomedical Engineering and Science (IJBES), 2012, pp 1-6

17. D.V.Becker, "Society of Nuclear Medicine Procedure Guideline for Thyroid Scintigraphy", 1999

18. V.Gupta, "Physiopathologic Atlas of Thyroid Scintigraphy", Kathmandu University Medical Journal, 2007, Vol. 5, pp 583-590

19. J.C.Bezdek, "Pattern Recognition with Fuzzy Objective Function Algorithms",PlenumPress. 1981 


\section{Morphological Reconstruction Filters}

Image Segmentation Techniques Using Pattern Recognition”, Medical Physics.1993,Vol. 20, pp.1033-1048

21. J.Serra, "Image Analysis and Mathematical Morphology", Academic press, 1982

22. R.C.Gonzalez, R.E.Woods R. E and S.L Eddins, "Digital Image Processing", $3^{\text {rd }}$, Pearson Education International, 2007

23. C.Y.Chang, H-C Huang and S-J.Chen, "Thyroid Nodule Segmentation and Component Analysis in Ultrasound Images", Proceedings in Asia-Pacific Signal and Information Processing Association, Japan. 2009, pp 910-917

24. C-Yu Chang, Y-F Lei, C-H Tseng and S-R Shih, "Thyroid Segmentation and Volume Estimation in Ultrasound Images", IEEE Transactions on Biomedical Engineering, 2010, Vol.57, pp.1348-1357

25. J.Kaur, A.Jindal, "Segmentation Algorithms for Thyroid Scintigraphy Images", International Journal of computer science and Technology, 2012, Vol.3, pp 449 -451. 